\title{
Multidimensional Scaling of Color Similarity in Bees
}

\author{
W. Backhaus, R. Menzel, and S. Kreiß1 \\ Institut für Tierphysiologie, Neurobiologie, Freie Universität Berlin, Königin-Luise-Strasse 28-30, D-1000 Berlin 33, Germany
}

\begin{abstract}
A multiple choice experiment with free flying bees trained to a color signal is described which allows for multidimensional scaling of color similarity. The choice proportions are analysed by metric (Torgerson 1958) and non-metric (Kruskal 1964a, b) multidimensional scaling. The light reflected from the twelve color signals used differed in spectral composition, intensity, and the proportion of white light. Only two scales are necessary to reconstruct the experimental data. The interpretation of the scale values by Helmholtzcoordinates, derived from the chromaticity diagram for bees, shows that the main perceptual parameters are hue and saturation (or blue/greenness and $\mathrm{UV} /$ blue-greenness, respectively). Brightness is ignored by the bees in this choice situation. The total color difference is related to the differences on the two perceptual parameters by the city-block metric (Minkowski exponent $p=1$ ).
\end{abstract}

\section{Introduction}

Multidimensional scaling (reviews Ahrens 1974; Davison 1983) has been used successfully in human psychophysics for the determination of the dimensions and the metric of color perception (Torgerson 1951; Kruskal 1964a; Indow and Ohsumi 1972). Human color vision is Euclidean and three dimensional. The perceptual parameters are interpretable as blue/yellowness, red/greenness, and brightness dimension (Helm 1964). The subjective color orders derived from the scale values are very similar to the Munsell color system, which gives the subjective order of colors with respect to hue, saturation and brightness. Bees have a trichromatic color vision system to which the Graßmannian laws apply, as Daumer (1956) showed with mixing experiments. In dual choice tests, bees ignore differences (up to $1: 4$ ) in intensity of two color signals if they are trained to spectral lights (Daumer 1956; Helversen 1972). However, bees are able to discriminate intensity differences, as shown by their spontaneous phototactic choice behavior with respect to spectral lights of different intensities $I(A I / I=0.135$ for a threshold discrimination of $65 \%$ in a dual choice test, and a reference light intensity of $3.6 \mathrm{~W} / \mathrm{m}^{2}$; Labhart 1974; see also Menzel and Greggers 1985, for wavelength dependence and absolute sensitivity). This means bees are able to discriminate the intensity of two lights significantly if the lights differ in intensity by $13.5 \%$. Helversen (1972) measured the threshold increments for wavelength $(\lambda)$ discrimination in bees with spectral lights $(\Delta \lambda=4.5 \mathrm{~nm}$ at $490 \mathrm{~nm}$ for $70 \%$ correct choices in a dual choice test). Lieke (in press) trained bees to white light and showed that they discriminate it very well from a mixture of white $\left(I_{w}\right)$ and seven different spectral lights $I_{\lambda}\left[I_{\lambda} /\left(I_{\lambda}+I_{w}\right)=(1-12) \%\right.$ for $62 \%$ correct choices in a dual choice test].

As in other animals, color perception in bees can be analysed only indirectly from the frequencies in choice experiments. The choice proportions can be taken as measure for color dissimilarity, because bees can easily be trained to color signals and are highly motivated in color choice experiments. Their color choice behavior is constant in time; the variance of the proportions estimated in a large series of tests do not differ from the variance of a binomial distribution (Helversen 1972). They also choose the trained color up to $100 \%$, if the alternative is different enough. If bees are trained in the reverse experiment to the alternative color signal, the choice proportions are nearly the same, although small asymmetries exist (Daumer 1956; Menzel 1967; Helversen 1972). Larger asymmetries, however, exist in the violet-blue region (Werner and Menzel 1984). In our experiment we worked with twelve simultaneously presented color signals in the blue-green region where the choice proportions are nearly symmetric. 
The adequate experimental and mathematical method to investigate color similarity relations in animals and humans is the method of triads (Torgerson 1958). One color signal of a triple of signals (triad) is trained and the other two are tested in a dual choice experiment. The method of triads means that every one of $n$ color signals is trained, and the remaining are tested in $n(n-1)(n-2) / 2$ dual choice experiments; i.e., 660 tests are needed for twelve colors. However, the results of dual choice experiments are also derivable from a multiple choice experiment (Guilford 1937). We tested this for bees by direct comparison of the derived dual choice proportions with proportions measured in dual choice experiments. This reduces the data collecting time drastically; twelve color stimuli can be investigated by twelve simultaneous tests. With his method of triads, Torgerson (1958) gave an extension of Thurstone's (1927) law of comparative judgment (Case V). Torgerson postulated that the proportions measured in a triadic experiment correspond to the difference in the perceptual distances between the trained color signal and the color signals of the pair tested. In accordance to Thurstone's one dimensional "Case V", the difference of the two perceptual distances compared is assumed to be the probability transform $[z$-value $(2,3)]$ of the measured proportions. The method of triads determines the $n(n-1) / 2$ comparative distances between $n$ stimuli from the $n(n-1)(n-2) / 2$ observed corresponding proportions by a least square solution. Since each of the distances is $n-2$ times over determined, the method of triads compensates experimental errors.

The matrix of comparative color distances is analysed by metric and non-metric scaling procedures (Torgerson 1958; Kruskal 1964a, b) to find the metric and the number of dimensions of the perceptual space which allows to calculate the color distances from the derived perceptual scales.

\section{Methods}

\subsection{Experimental Arrangement}

A vertically arranged round plate $(\varnothing 1.2 \mathrm{~m})$ was mounted with its centre axis ( $1.5 \mathrm{~m}$ above ground) on a stand in such a way that it could be rotated. A bee was trained to three identical color signals in three fixed positions with different distances from the centre of the plate (Fig. 1). The plate was rotated around its axis in several positions in order to train the individually marked bee to the color signal independently from the position of the signal. In the test situation the twelve color signals in three groups of four were presented simultaneously. The three small round plates and the

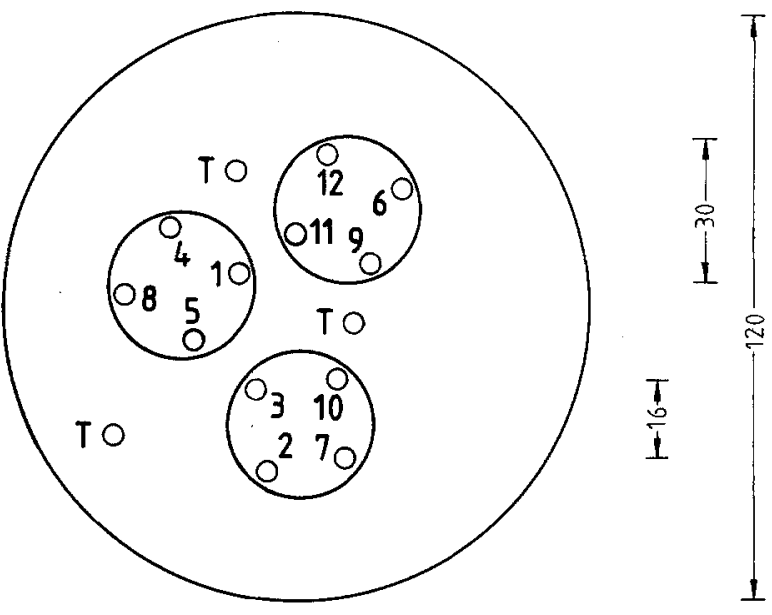

Fig. 1. Frontal view of the apparatus for multiple color choice experiments. The numbers indicate the color signals (see Fig. 2). During training the rewarded color signal $T$ appears on three places outside the three groups of four color signals, and the one place of the trained color signal within one of these groups is covered by a disc of the same grey as the background. At the beginning of a test the three training color signals $T$ are covered, the one within the group of four color signals uncovered, and the configuration is changed by rotating the big plate and the three small plates. The background screen and the three small plates are painted evenly grey

large plate were rotated between the tests such that the absolute and the relative positions of the twelve signals were changed.

The color signals were round discs $(\varnothing 50 \mathrm{~mm})$. Ten of the twelve signals used in the experiment were pigment paint on cardboard and two were glass filters (Shott and Gen., Mainz) on top of a sanded aluminium disc (see Menzel and Lieke 1983 for further details on the experimental arrangement). We chose the color signals such that they varied in their physical parameters over a wide range in spectral, white light, and total reflectance. On the other hand, we ensured in preliminary experiments that the chosen set does not appear too different to the bees, by testing the color signals most different in their physical parameters (Table 1). The photon fluxes absorbed by the three receptors of the bee from the photon flux of the twelve color signals are presented as loci of color stimuli in the chromaticity diagram (Fig. 2). We assume, that the receptors are adapted to the background light $B$ in the center point. If the bee is adapted to daylight, the center point lies little left from stimulus 2 .

\subsection{Training}

A group of bees (Apis mellifera) was trained from the hive $20 \mathrm{~m}$ south of the apparatus. These bees recruited new bees by their dances. When a new bee arrived, it was marked as the experimental bee and all others 
Table 1. Proportions for the discrimination case (one of the color signals in the pair is the trained one) derived from the multiple choice experiment as a test on symmetry. The numbers denote the color signals (see Fig. 2). The difference between the discrimination proportions and those of the reverse experiment is rarely larger than 0.12

\begin{tabular}{r|llllllllllll|}
\multicolumn{1}{l}{} & \multicolumn{1}{l}{} & $\mathbf{2}$ & $\mathbf{3}$ & $\mathbf{4}$ & $\mathbf{5}$ & $\mathbf{6}$ & $\mathbf{7}$ & $\mathbf{8}$ & $\mathbf{9}$ & 10 & 11 & 12 \\
\hline & & 0.58 & 0.64 & 0.68 & 0.57 & 0.57 & 0.65 & 0.74 & 0.59 & 0.65 & 0.61 & 0.74 \\
2 & 0.61 & & 0.82 & 0.74 & 0.60 & 0.66 & 0.74 & 0.79 & 0.76 & 0.82 & 0.72 & 0.79 \\
3 & 0.68 & 0.76 & & 0.51 & 0.65 & 0.55 & 0.56 & 0.65 & 0.59 & 0.57 & 0.48 & 0.60 \\
4 & 0.71 & 0.79 & 0.62 & & 0.61 & 0.58 & 0.59 & 0.65 & 0.66 & 0.69 & 0.63 & 0.81 \\
5 & 0.73 & 0.75 & 0.80 & 0.59 & & 0.51 & 0.55 & 0.63 & 0.70 & 0.82 & 0.77 & 0.82 \\
6 & 0.79 & 0.84 & 0.66 & 0.64 & 0.68 & & 0.57 & 0.61 & 0.89 & 0.80 & 0.71 & 0.86 \\
7 & 0.79 & 0.83 & 0.64 & 0.64 & 0.71 & 0.50 & & 0.59 & 0.86 & 0.79 & 0.69 & 0.73 \\
8 & 0.87 & 0.95 & 0.74 & 0.74 & 0.83 & 0.67 & 0.65 & & 0.92 & 0.88 & 0.80 & 0.69 \\
9 & 0.77 & 0.84 & 0.75 & 0.74 & 0.76 & 0.82 & 0.87 & 0.90 & & 0.62 & 0.67 & 0.83 \\
10 & 0.67 & 0.76 & 0.64 & 0.64 & 0.66 & 0.74 & 0.81 & 0.84 & 0.38 & & 0.55 & 0.74 \\
11 & 0.77 & 0.88 & 0.59 & 0.66 & 0.71 & 0.69 & 0.72 & 0.72 & 0.55 & 0.58 & & 0.60 \\
12 & 0.89 & 0.94 & 0.74 & 0.81 & 0.90 & 0.90 & 0.84 & 0.74 & 0.81 & 0.70 & 0.67 & \\
\hline
\end{tabular}

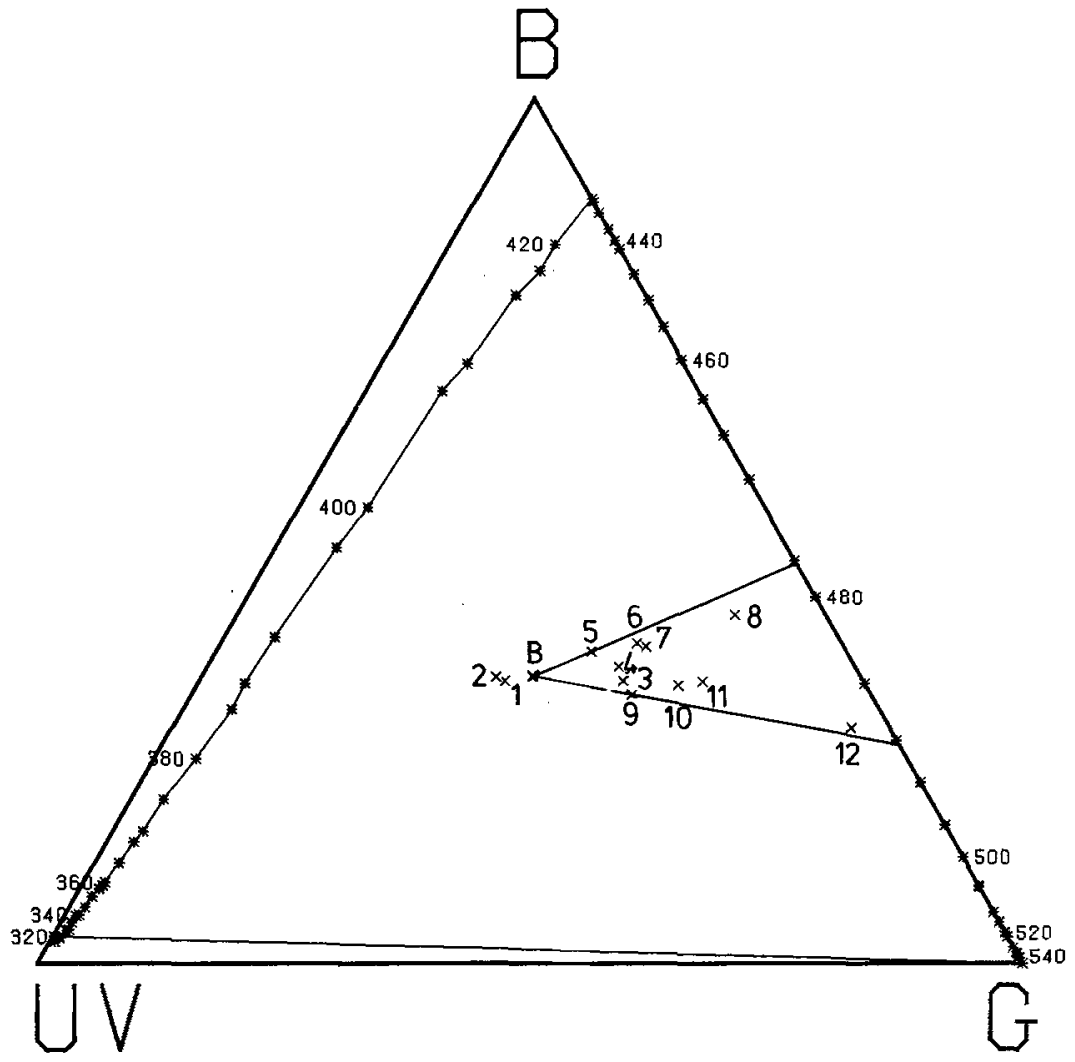

Fig. 2. Chromaticity diagram of the bee. The numbers denote the color stimuli which are defined by the relative numbers of photons absorbed by each of the receptors per unit time. The corresponding color signals illuminated by daylight used in our experiment were: $1:$ Alu $+\mathrm{F}$ 2: Gray 3: BV1 4: BV2 5: BV3 6: BV3 +F 7: BV3 +2F 8: BG18 9: GR4 10: GR4+F 11: GR4+2F 12: VG6. $F$ denotes a gray foil which decreases in principal total reflection. The color signals were covered with UV transparent plexiglas except the two glass filters 8,12 were kept in a cage. The experimental bee was fed dropwise with sucrose solution from a silicone pipe in the middle of the color signal to which it was trained. The bee received less than $10 \mu \mathrm{l}$ at each landing; therefore, it had to fly up 5-10 times to the other training signals until it was satiated. After 6-8 visits at the apparatus (30-80 learning acts), the bees' learning reached an asymptode and could be tested. We obtained an asymptotic level of acquisition of $90 \%-95 \%$ choice proportion. The bees were either trained with or without the other twelve color signals present during training (called absolute or differential training, respectively). Comparison of the choice proportions should help to analyse the effect of the training method.

\subsection{Tests}

The bees were not fed during the tests and the three training signals were covered with grey discs. In order to maintain high motivational levels, all tests were limited to three minutes, after which the bee was fed 
again as described above. Tests were repeated 6-10 times. The bee was then taken away from the experiment and was marked as a normal collector bee. A videosystem recorded the choice behavior and the experimenter recorded direct approaches towards a color signal, touching it or landing on it, as a choice. The tests were performed in two ways: multiple choice tests were those with all twelve color stimuli presented simultaneously, and dual choice tests with only two color signals, the trained one together with one out of the other twelve alternatives.

\section{Results}

\subsection{Results of the Behavioral Experiments}

Bees choose the color signals homogeneously across the round plate. Figure 3 a shows the flight trace of a three minutes record. The choices of the color signals were so obvious to the experimenter that we gave up the video recordings after we had established firmly that a simultaneous protocol by the experimenter gave the same results as a blind evaluation of the video tapes. In Fig. $3 b$ the loci of a bee drawn every second during three tests after it was trained to color signal 10 is presented. Though the trained color signal lies in the lower half of the plate during the three tests, the bee searches homogeneous across the whole plate. The results of all multiple choice tests are given as relative choice frequencies in Fig. 4.

Next we studied the question whether the different training methods (absolute vs. differential training)
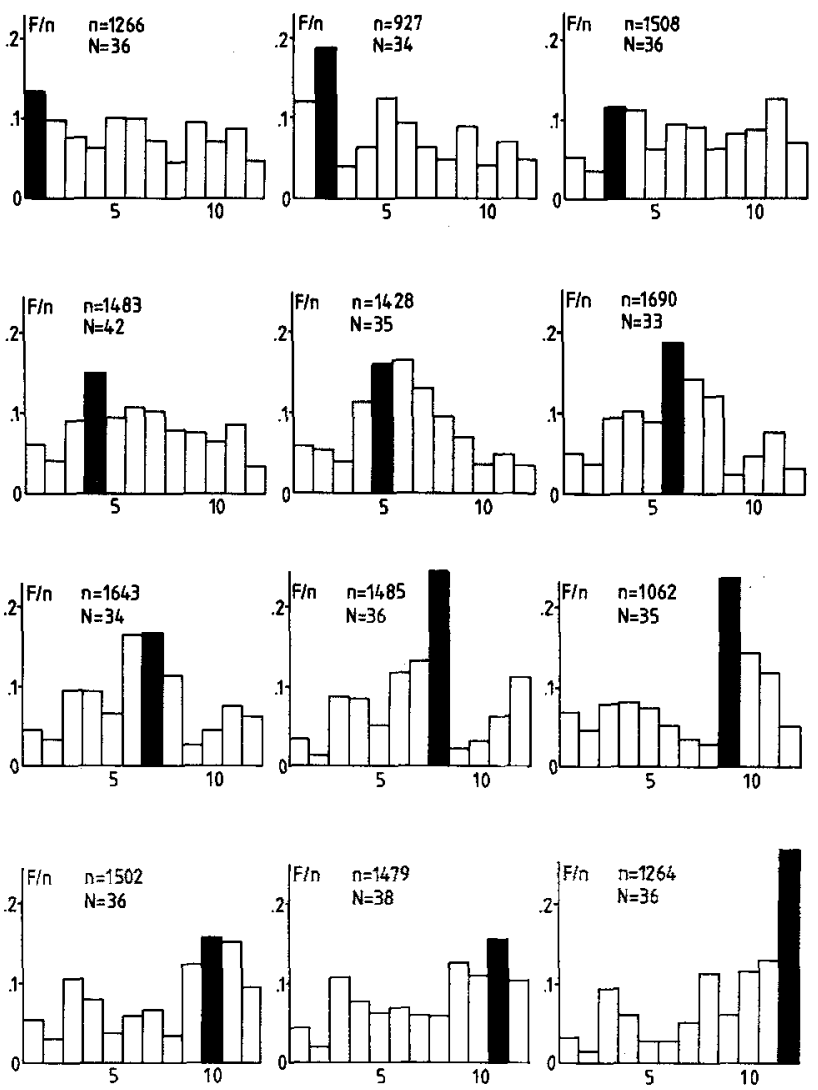

Fig. 4. Relative choice frequencies $F_{i} / n$ of all multiple tests. The number of the color stimuli is given on the abscissa. The black bar indicates the color stimulus trained. $N$ gives the number of tests with the total number of choices $n$. Color signals $1-3$ and 5-12 were trained to 6 bees each, signal 4 was trained to 7 bees

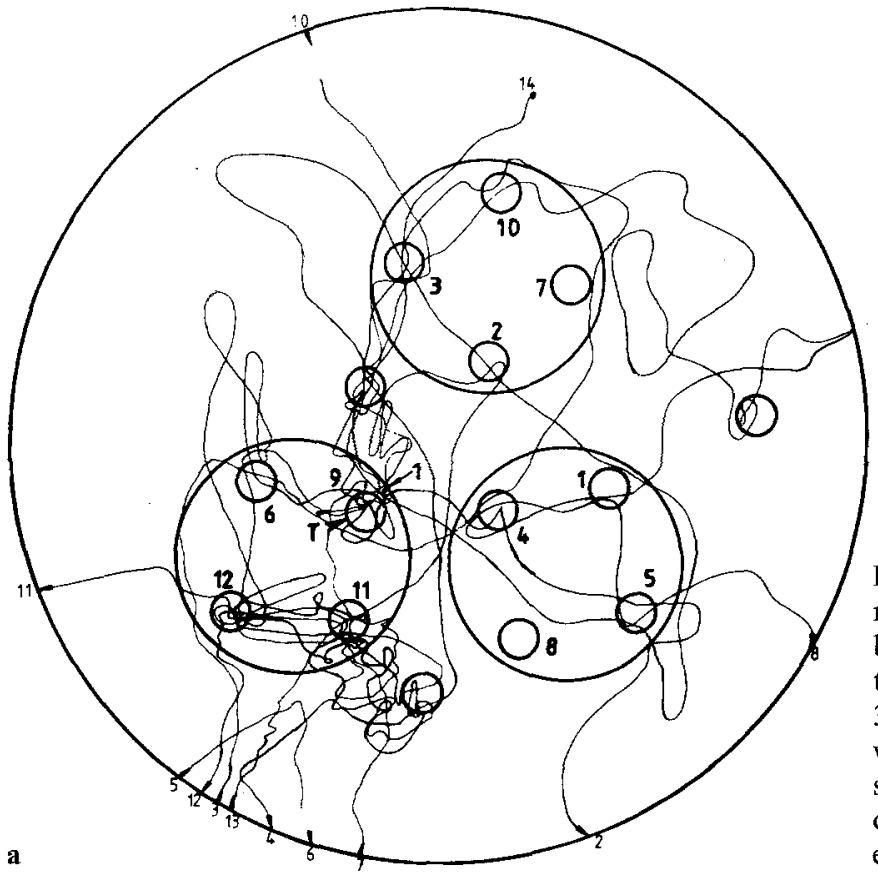

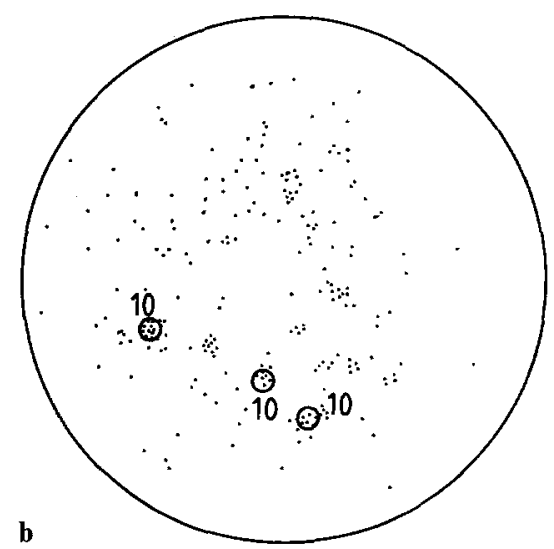

Fig. 3. a Typical flying trace of a bee reconstructed from video recordings. The bee was trained to color signal 9. The choice behavior of the bee in this particular test was estimated on-line by the experimenter as follows: color signal $1: 0,2: 2,3: 3,4: 2,5: 3,6$ : $3,7: 1,8: 0,9: 5,10: 1,11: 6,12: 3$. b A dot chart of three tests in which the position of the bee is indicated by a dot for every one second interval. The positions of the trained color signal 10 during the three tests are represented. The bee flight is homogeneous across the plate 
had any influence on the choice behavior. We did not find any statistically significant differences (comparison of $95 \%$ confidence intervals) although there is a trend that relatively similar colors are somewhat better discriminated after differential training than after absolute training. These small differences have not been analysed further here, and are considered to be of little or no influence. The results of experiments with differential and absolute training were pooled.

Multiple choice data have to be transformed into dual choice values if multidimensional scaling methods are to be applied. We therefore tested repeatedly whether the choice behavior depends on the test procedure for several pairs of color signals, in dual (one alternative) or multiple (eleven alternatives) choice tests. The choice frequencies of the multiple tests are converted into the corresponding dual choice proportions by dividing the frequencies for the trained color signal or an alternative respectively, by the sum of these frequencies and the frequencies of the particular alternative in turn (1). Figure 5 shows the comparison of all dual choice proportions with the corresponding dual choice proportions calculated from multiple choice tests. The scatter bars give the $95 \%$ confidence intervals. No statistically significant differences in the range of validity $\leqq 0.05$ are found between the two test procedures. We conclude, therefore, that dual choice frequencies calculated from the choice values of multiple choice tests with bees are usable for multidimensional scaling. Furthermore, the color differences are 10 times overdetermined (see above).

We tested the symmetry of the choices by the calculated choice proportions corresponding to discrimination tests, where one of the tested color signals was the trained one. Close inspection of the reciprocal choice proportions listed in Table 1 shows that most of the results of the reverse tests were symmetrical within the scatter of the data $(0.06)$ and only few (e.g. 9 vs. 10 , 9 vs. 11,5 vs. 8,3 vs. 5,2 vs. 6 ) are just above a difference of 0.12 to the mean proportions. These few asymmetries do not affect the color distances much, because of the 10 times overdetermination (for the calculation of color distances see (6)).

\subsection{Determination of Color Distances}

As an experimental result from the multiple tests, we obtained twelve choice frequencies for each of the twelve trained color stimuli. From these choice frequencies $\mathbf{F}$, twelve $12 \times 12$ matrices $\hat{\mathbf{P}}$ of equivalent dual choice proportions with respect to the trained color (triadic test/train combination) are calculated according to the method of choices (Guilford 1937):

${ }_{k} \hat{p}_{i j}={ }_{k} f_{i} /\left({ }_{k} f_{i}+{ }_{k} f_{j}\right) ; \quad i, j, k=1, \ldots, n=12$.
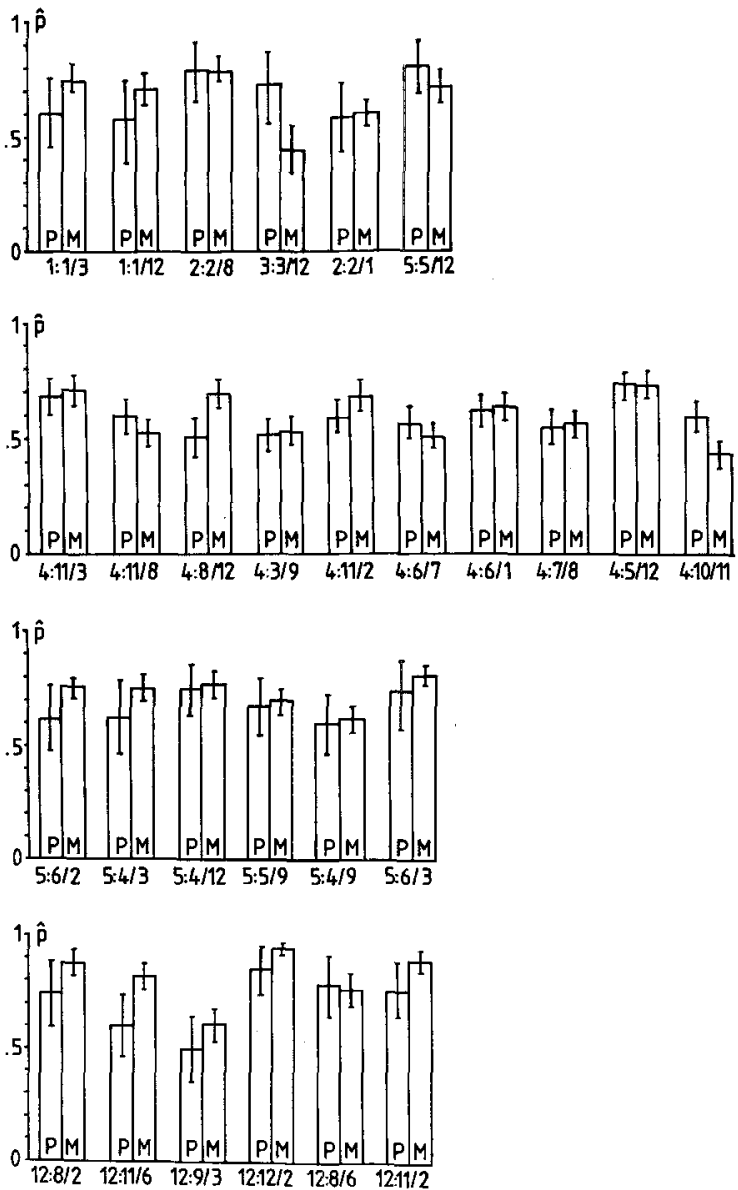

Fig. 5. Comparison of the dual choice proportions $\hat{p}$ measured in pair comparison experiments $(P)$ with the dual choice proportions calculated from the choice frequencies of the multiple choice tests $(M)$ (Fig. 4). The abscissa gives the triads tested: The number of the trained stimulus is given in front. The two numbers behind the colon give the signal combination tested. The proportions correspond to the signal number left from the slash

The element ${ }_{k} \hat{p}_{i j}$ is the observed proportion of times color $i$ was judged more similar to the trained color $k$ than color $j$ was judged. In the following, elements of matrices and vectors are denoted as small letters with indices. Small letters without index denote constants.

Assuming the choice behavior of the bee to depend exclusively on the color signals, the choice proportions can be taken as measure for the difference of the dissimilarity between the trained color $k$ and the two alternatives $i, j$ in test. In order to get a linear measure according to the law of comparative judgment (Case V) (Thurstone 1927) the choice proportions are linearised by probability transformation into $z$-values which are defined by the inverse of the normal distribution function

${ }_{k} p_{i j}=1 /(2 \pi)^{1 / 2} \int_{-\infty}^{k^{z_{i j}}} \exp \left(-1 / 2 z^{\prime 2}\right) \mathrm{d} z^{\prime}$ 
approximated (modified after Zielinski 1978) with an error $<1 \%$ by:

${ }_{k} z_{i j}=1 /(8 \pi)^{1 / 2} \ln \left({ }_{k} p_{i j} /\left(1-{ }_{k} p_{i j}\right)\right), \quad 0<{ }_{k} p_{i j}<1$.

The dissimilarity of colors is representable as distances $\left(d_{k i}\right)$ in a multidimensional metric (perceptual) space (Minkowski space) (Krantz 1967). The $z$-values, which are measured as choice proportions by the method of triads (Torgerson 1958), are interpreted as the difference of the distances:

${ }_{k} z_{i j}=d_{k j}-d_{k i}$.

The true distances $d_{i j}$, representing the similarity between two colors, are determined as comparative distances $h_{i j}$ only up to an additive constant $c$ which is not measurable, because $c$ is subtracted in (4):

$d_{i j}=h_{i j}+c$.

Calculating $h_{i j}$ from (5) in connection with (4) with different $z$-values, it is seen, that $h_{i j}$ is $n-2$ times overdetermined. The best estimate for elements of the matrix $\mathbf{H}$ of comparative distances is derived from the $z$-values as a least square solution:

$h_{i j}=\left({ }_{i} z_{\cdot j}+. z_{\cdot i}+{ }_{j} z_{\cdot i}+. z_{\cdot j}\right) / 2$.

The indices in front of the elements of $\mathbf{Z}$ indicate the trained color signal, the two indices behind denote the tested pair of color signals. The dot symbolises that all elements of $\mathbf{Z}$ with this index have to be averaged.

\subsection{Multidimensional Scaling of Color Similarity}

The models of multidimensional scaling (MDS) suppose the distances $d_{i j}$ between two colors to be derived by a composition rule (metric) from the differences on $d$ independent perceptual dimensions (scales) of a metric space (Minkowski space). The multidimensional scaling procedures determine these components in different dimensions with different metrics. From the Minkowski spaces tried, that one is taken as a mathematical representation of subjective color similarity which allows a good reproduction of the measured comparative distances $h_{i j}$ (metric MDS) or the rank orders of $h_{i j}$ (non-metric MDS). The number of dimensions is kept as small as possible. The goodness of reproduction is determined by error measures (residua, stress) involved in the procedures used.

Two different procedures are available. The metric procedure of Torgerson (1958) and the non-metric procedure of Kruskal (1964a, b). The metric procedure solves the scaling problem exact in a Euclidean vector space. The comparative distances are expressed as scalar product between every two distance vectors from the trained color to the two tested colors. Since scalar products have the mathematical properties of correlation coefficients, it is possible to factorise the matrix of scalar products, i.e. the minimum number of scales is determined which allows to reconstruct the matrix of scalar products. The scale values give the magnitude of the perceptual color components (attributes) caused by the corresponding color stimulus (see Appendix I).

The non-metric procedure is an iterative procedure which extends multidimensional scaling to Minkowski metrics. The procedure begins with a starting configuration of scale values and tries to fit the rank orders of all measured distances $d_{i j}$ by changing the configuration step by step, calculating the distances from a Minkowski metric and determining the rank orders. The changes of the scale values are performed in the direction of steepest decent of the goodness of reproduction function (stress) until it becomes a minimum. Though the procedure only compares the rank orders of the distances, the results are metric scales (see Appendix II).

We applied both procedures to the distances measured in our experiment. All formulas used are given in the Appendices I, II. The mathematical operations are explained, so far it is necessary to understand the main steps of the procedures. Mathematical details can be found in the original publications of Torgerson (1958) and Kruskal (1964a,b).

A flow chart of the application of metric (Appendix I) and non-metric (Appendix II) procedures to our results of the multiple choice measurement of twelve color stimuli is given in Fig. 6. First the comparative distance matrix $\mathbf{H}$ is derived from the measured choice frequencies $\mathbf{F}$ as described in Sect. 3.2. Then the metric procedure starts to estimate the number of dimensions $d$ and to get a reasonable starting configuration for the non-metric procedure, better than a random one. The Messick and Abelson solution is used for the estimation of the additive constant (Messick and Abelson 1956, see Appendix I). A typical scatter plot of the original distances and the distances derived from the scales computed in two dimensions is shown in Fig. 7. The scales of the metric solution in two to four dimensions are then used as input for the non-metric procedure. For the one dimensional case there is no difference between the metrics with different exponents $p$.

Our computer programs (Fortran 77, Cyber 835) realised the numerical algorithms of the following authors: 1) method of triads and metric MDS, Torgerson (1958), 2) Jacobi algorithm for eigenvalues and eigenvectors, Nash (1979), 3) gradient method, Kruskal $(1964 \mathrm{a}, \mathrm{b})$; a part of program $(G)$ was implemented from Sixtl (1982).

We checked our programs by model data taken from Torgerson (1958) and Sixtl (1982). Since matrix 
Multidimensional Scaling of Similarity

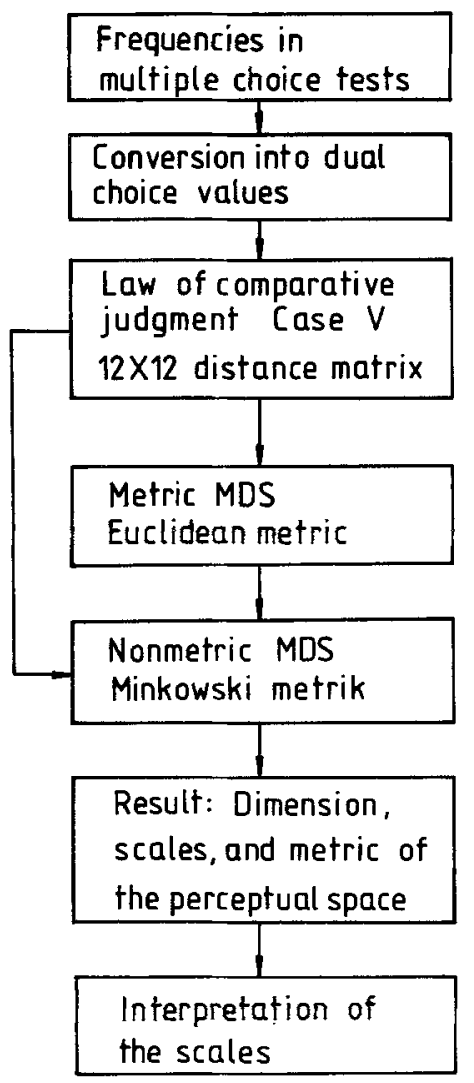

J.P. Guilford

L.L. Thurstone

W.R. Torgerson

W. R. Torgerson

J.B. Kruskal

Fig. 6. Flow chart of the application of metric and non-metric multidimensional scaling procedure to the choice frequencies of the multiple choice experiment (see text and Appendices I, II)

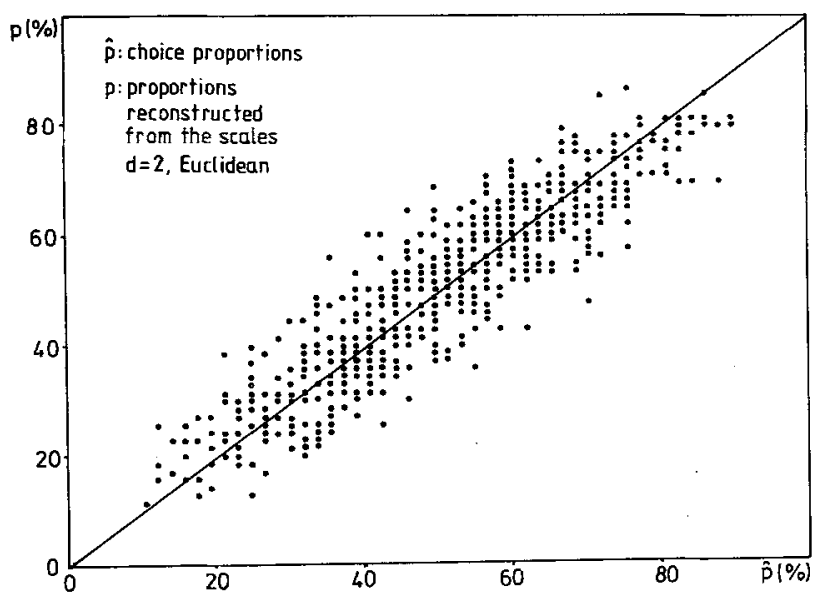

Fig. 7. Scatterplot of the original proportions $\hat{p}_{i j}$ (abscissa) and the proportions $p_{i j}\left(z_{i j}\right)$ (ordinate) derived from the scales of the metric procedure in two dimensions from (16) (see Appendix I). Since the sum of the corresponding elements of the upper and the lower half of matrix $\mathbf{Z}$ is always 1, only the proportions of the upper half are presented to test the scatter on homogeneity calculations are very sensitive to the precision of small numbers, accuracy of the results was tested by double precision runs. No difference to single precision runs was found up to the order of $10^{-10}$. It turns out that care has to be taken with the convergence criterion in the gradient method of Kruskal. By testing the convergence behavior of the $S$ function with Kruskal's parameters in the gradient method applicated to our data we found a robust criterion for the absolute convergence to be that the last five values of the $S$ function have not changed by more than $10^{-5}$.

\section{Discussion and Conclusion}

The normalized stress $S$ is plotted against the Minkowski exponent $p$ in Fig. 8. Since $S$ is the measure for the goodness of reproduction of the measured distances by the model distances (small values indicate a good, big values indicate a bad reproduction), it can be seen from Fig. 8 that the Euclidean solution $p=2$ is worse than all the other solutions. The minimum of $S$ lies at the extremes $p=1-1.2$ (city-block metric) and $p=40$ (dominance metric). In Fig. $9 S$ is plotted against the number of dimensions $d$ for the typical cases $p=1$, $p=2, p=40$. The goodness of reproduction function $S$ bends at $d=2$. The value of $S$ is beyond 0.1 , which indicates a good level of reproduction. The solution for $d=3$ leads to a value of $S$ below 0.05 . Following the guidelines of Kruskal and Wish (1978) it is rarely necessary to add dimensions that reduce the value of $S$ below 0.05 .

As it can be seen by comparison of the perceptual color configurations, the dominance solution $(d=3$, $p=40$ ) (Fig. $10 \mathrm{~b}$ ) looks close to the city-block solution $(d=2, p=1)$ (Fig. 10a) when the configuration of the dominance solution is rotated by ca. $45^{\circ}$ clockwise around the center-point. The accuracy analysis of the scale values was performed by a Monte-Carlo-simulation. In addition to the measured proportion matrix $\mathbf{P}, 100$ proportion sets were generated randomly according to the Gaussian distributed empirical error (from the proportions per test) interval of the measured proportions. In 100 runs the generated proportions were taken as input for the metric procedure to calculate the scale values of the error range. The error bars drawn in Fig. 10a at color 9 represent the most probable error of the values calculated from the measured proportions. All other error bars (not drawn) are of the same order. This shows that the accuracy of multiple color choice experiments is good enough for multidimensional scaling of color similarity in agreement with an earlier performed error estimation by Monte-Carlo-simulation (Backhaus and Menzel 1984). 


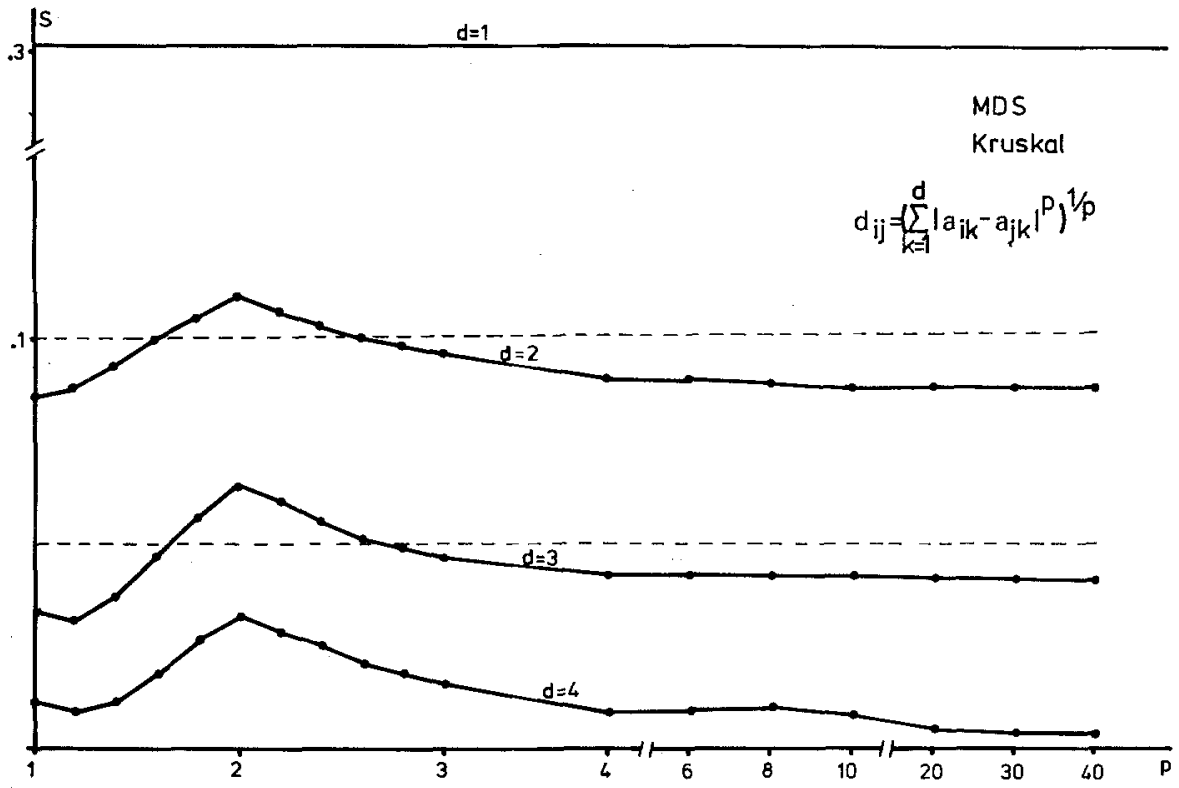

Fig. 8. Goodness of reproduction function (normalized stress) $S$ depending on the metric parameter $p$ (see text)
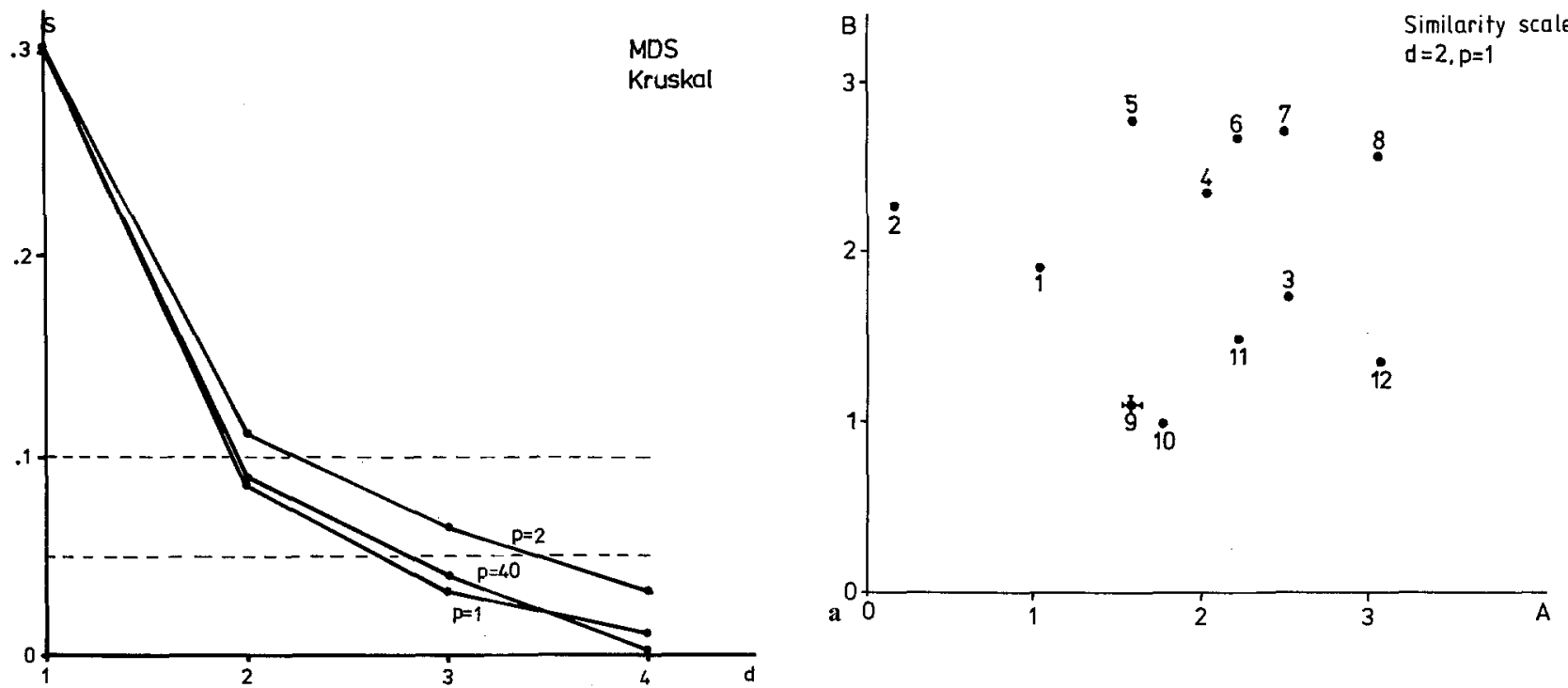

Fig. 9. Goodness of reproduction function (normalized stress) $S$ depending on the dimension $d$. Selection of the three most important cases from the data shown in Fig. 8: city-block metric $p=1$, Euclidean metric $p=2$, dominance metric $p=40$

Fig. 10. a Result of non-metric multidimensional scaling: The two color similarity scales $A$ and $B$ for the case $d=2$ and cityblock metric $(p=1)$. The scales are a measure for color dissimilarity between the twelve colors as they appear to the bee. This subjective arrangement of colors is topologically similar to the arrangement of color stimuli in the chromaticity diagram (see Fig. 2). b The corresponding scales $A$ and $B$ for the case $d=3$ and dominance metric $(p=40)$

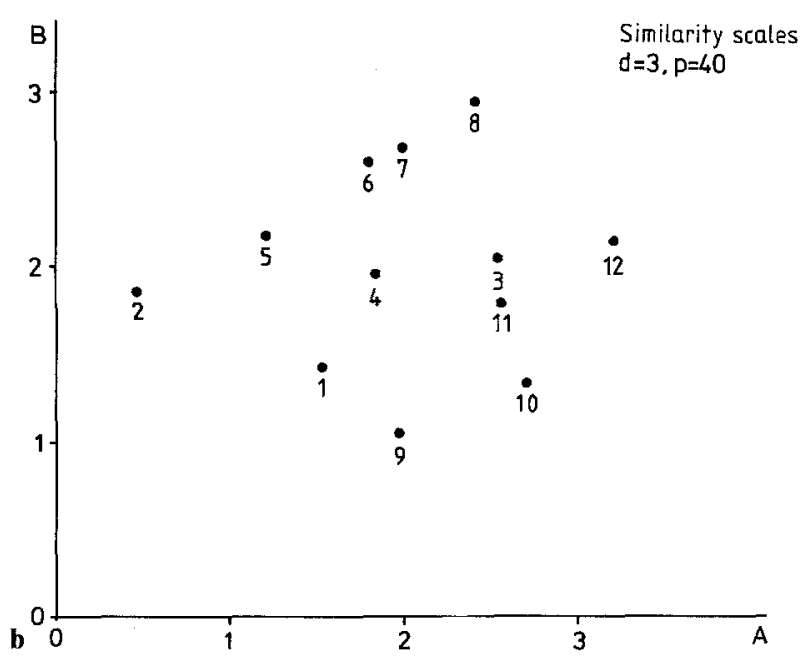


Table 2. Helmholtz-coordinates of the used color stimuli. Hue $H(\mathrm{~nm})$ and saturation $S(\%)$ are derived graphically from the chromaticity diagram (Fig. 2). Brightness is calculated as the sum of the absorbed photon fluxes by the receptors and is expressed relatively to the brightness of the background

\begin{tabular}{c|c|c|c|c|c|c|c|c|c|c|c|c|}
\hline & 1 & 2 & 3 & 4 & 5 & 6 & 7 & 8 & 9 & 10 & 11 & 12 \\
\hline $\mathrm{S}$ & .08 & .13 & .25 & .28 & .22 & .37 & .40 & .72 & .27 & .41 & .51 & .87 \\
\hline $\mathrm{H}$ & 6. & 6. & 3.5 & 6 & 12. & 9.5 & 8. & 9. & 1. & 3. & 4. & 1. \\
\hline $\mathrm{B}$ & 1.78 & 1.67 & 1.09 & 1.77 & 3.36 & 2.15 & 1.67 & .85 & 2.33 & 1.2 & 1.01 & .22 \\
\hline
\end{tabular}

It is open to interpretation which set of scales gives the better representation for color perception in bees. Since there exists no subjective color order system like the Munsell system for humans, and since we know that bees can discriminate spectral lights from each other as well as white light from mixtures of white and spectral light, we take the Helmholtz-coordinates hue, saturation and brightness for the color stimuli as possible estimates for the subjective order in three dimensions (Table 2). Another interpretation would be blue/greenness, UV/blue-greenness, and brightness. A quantitative interpretation of the two scales as excitations of color opponent channels should be possible when the mechanisms of color opponency in bees have been worked out, which will be our next goal.

Hue is estimated graphically as absolute difference in wavelength from the chromaticity diagram for bees (Fig. 2, see also Backhaus and Menzel 1987). Saturation is calculated as the mixture coefficient assuming the color stimuli to be mixed from white and spectral light. Brightness is assumed to be equal to the sum of the tristimulus values of the color stimuli.

Let us first investigate the derived scales by correlation analysis. Between all possible combinations of Helmholtz-coordinates and scales, the ordinary and the partial correlation coefficients are calculated. From Table 3, we see: 1 . The Helmholtz-coordinates of the used color stimuli show an anticorrelation between saturation and brightness and a correlation between hue and brightness. Hue and saturation show no significant correlation. 2 . The three derived scales are orthogonal. 3. The scales for $p=1$ in the two dimensional case are interpretable as saturation and hue parameters. Brightness shows the same bad correlation to scale $A$ as to scale $B$. 4. The scales of $p=40$ for two dimensions show a bad correlation to brightness, too. The correlation coefficients do not differ much from the case $d=2, p=1$. This is reasoned in the rotation of ca. $45^{\circ}$ of the two configurations relative to each other (see above). 5 . When we investigate the three dimensional case, we get the same results. Scale $C$ is not interpretable and thus not necessary.

In case of three dimensions, it is possible that the three scales are not simply connected to a total color
Table 3. Correlation analysis. The upper numbers denote the ordinary and the lower numbers denote the partial correlation coefficients.

Top row: The correlation matrix of the Helmholtz-coordinates of the color stimuli and the scales of the three dimensional case for $p=1$.

Middle rows: Interpretation of the derived scales in two and three dimensions with $p=1$ and $p=40$ by the Helmholtzcoordinates of the color stimuli. The correlation matrices show the goodness of interpretation.

Bottom row: Two possible cases of mixed metric in three dimensions. The goodness of reproduction function $S$ is worse than in the simple metric cases. The goodness of interpretation is worse, too. Brightness does not correspond to one of the three scales as in the cases of simple metric.

(Correlation coefficients $>0.71$ are different from random on the $1 \%$ level)

Correlationcoefficients

\begin{tabular}{|c|c|c|c|c|c|c|c|}
\hline \multicolumn{3}{|c|}{$\begin{array}{l}\text { Saturation, } \\
\text { Brightness }\end{array}$} & \multirow{2}{*}{$\begin{array}{l}\text { Hue, } \\
\text { B }\end{array}$} & \multicolumn{2}{|c|}{ Scales } & $\mathrm{d}=3$ & $\mathrm{p}=1$ \\
\hline & $\mathrm{s}$ & $\mathrm{H}$ & & & A & B & C \\
\hline & 1. & $\begin{array}{r}-.23 \\
.30\end{array}$ & $\begin{array}{l}-.70 \\
-.71\end{array}$ & A & 1. & $\begin{array}{r}.08 \\
-.07\end{array}$ & $\begin{array}{l}-.18 \\
-.18\end{array}$ \\
\hline & $\begin{array}{r}.23 \\
.30\end{array}$ & 1. & $\begin{array}{l}.58 \\
.60\end{array}$ & B & $\begin{array}{r}.08 \\
-.07\end{array}$ & 1. & $\begin{array}{l}-.04 \\
-.04\end{array}$ \\
\hline & $\begin{array}{l}-.70 \\
-.71\end{array}$ & $\begin{array}{l}.58 \\
.58\end{array}$ & 1. & C & $\begin{array}{l}-.18 \\
-.18\end{array}$ & $\begin{array}{l}-.04 \\
-.03\end{array}$ & 1. \\
\hline
\end{tabular}

Interpretation of the Scales

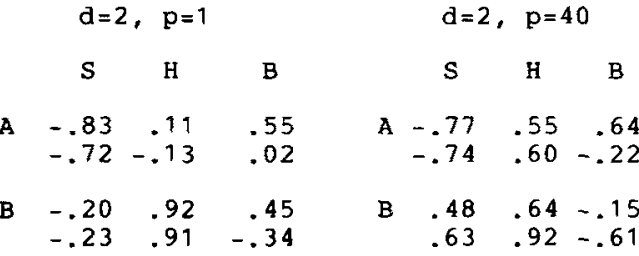

\begin{tabular}{|c|c|c|c|c|c|c|c|}
\hline & $d=$ & $\mathrm{p}=1$ & & & $d=3$ & $3, p=4$ & 40 \\
\hline & s & H & B & & $s$ & $\mathrm{H}$ & B \\
\hline & $\begin{array}{l}-.80 \\
-.62\end{array}$ & $\begin{array}{l}.06 \\
.34\end{array}$ & $\begin{array}{l}.60 \\
.28\end{array}$ & A & $\begin{array}{l}-.78 \\
-.67\end{array}$ & $\begin{array}{l}.54 \\
.49\end{array}$ & $\begin{array}{l}.72 \\
.05\end{array}$ \\
\hline$E$ & $\begin{array}{l}-.26 \\
-.15\end{array}$ & $\begin{array}{l}.93 \\
.90\end{array}$ & $\begin{array}{r}.55 \\
-.08\end{array}$ & B & $\begin{array}{l}.46 \\
.62\end{array}$ & $\begin{array}{l}.66 \\
.93\end{array}$ & $\begin{array}{l}-.14 \\
-.67\end{array}$ \\
\hline & $\begin{array}{l}.13 \\
.43\end{array}$ & $\begin{array}{l}-.11 \\
-.37\end{array}$ & $\begin{array}{l}.14 \\
.47\end{array}$ & C & $\begin{array}{r}-.08 \\
.36\end{array}$ & $\begin{array}{r}.03 \\
-.34\end{array}$ & $\begin{array}{l}.36 \\
.53\end{array}$ \\
\hline & $3, A: p=$ & $=40 ; B, C$ & $c: p=1$ & $d=$ & $3, c: p=$ & $=40 ; \mathrm{A}$, & $B: p=1$ \\
\hline & $\mathbf{s}$ & $\mathrm{H}$ & $\mathbf{B}$ & & $\mathbf{s}$ & H & B \\
\hline & $\begin{array}{l}-.79 \\
-.56\end{array}$ & $\begin{array}{r}.27 \\
-.07\end{array}$ & $\begin{array}{l}.70 \\
.31\end{array}$ & A & $\begin{array}{l}-.80 \\
-.66\end{array}$ & $\begin{array}{c}.0 \\
-.38\end{array}$ & $\begin{array}{l}.55 \\
.22\end{array}$ \\
\hline & $\begin{array}{l}.02 \\
.24\end{array}$ & $\begin{array}{l}.89 \\
.90\end{array}$ & $\begin{array}{r}.33 \\
-.23\end{array}$ & B & $\begin{array}{l}-.31 \\
-.21\end{array}$ & $\begin{array}{l}.93 \\
.90\end{array}$ & $\begin{array}{r}.59 \\
-.03\end{array}$ \\
\hline & $\begin{array}{r}-.07 \\
.27\end{array}$ & $\begin{array}{l}-.05 \\
-.34\end{array}$ & $\begin{array}{l}.27 \\
.44\end{array}$ & C & $\begin{array}{l}.56 \\
.61\end{array}$ & $\begin{array}{l}-.04 \\
-.13\end{array}$ & $\begin{array}{r}-.19 \\
.35\end{array}$ \\
\hline
\end{tabular}


difference by one metric but by a mixture of different metrics. Since step control of the iterative procedure is not designed for these more complicate cases, the procedure only converges for the case that $A$ and $B$ are connected by the city-block metric $p=1$, and $C$ only contributes if the total difference in $A$ and $B$ is smaller than the difference in $C$. The other case is, that $A$ dominates the total difference in $B$ and $C$ (cityblock metric). In both cases, the function $S$ is worse than in the normal three dimensional city-block case. Again, brightness is not interpretable with these solutions, too. If we would take UV/blue-greenness instead of saturation for the interpretation of the scales, the correlations should remain nearly the same; only the values for signals 1 and 2 will have little influence on the correlations. We cannot perform this interpretation quantitatively, because the excitation of a color-opponent system can not be calculated, yet. We are working on a color opponent model for bees at this time.

We conclude from these results that the bee ignores differences in brightness in color choice experiments of the kind used here, and rates differences in hue and saturation (or blue/greenness and UV/blue-greenness) high; this is consistent with the findings of Daumer (1956) and Helversen (1972). This result does not exclude that bees perceive brightness differences of spectral lights, e.g. in phototaxis experiments as shown by Labhart (1974) and Menzel and Greggers (1985). When they learn color signals at the feeding place, however, they make no use of the information about differences in brightness over a wide range. From the configuration $(p=1, d=2)$ shown in Fig. 10a and Table 1 we read that a difference of $5 \mathrm{~nm}$ in hue or a difference of $40 \%$ in saturation leads to a significant ( $70 \%$ right choices) difference in choice proportion, respectively. The difference between the colors 8 and 12 is judged nearly equal as the difference between the colors 5 and 9 . The colors 1 and 2 are judged as very different. This is in good agreement with the finding of Lieke (in press) who determined that bees are able to discriminate a mixture of white and spectral light very well from white light. The colors 4, 3, 11 lie very far to the right. This may indicate that saturation discrimination is hue dependent.

The experimental arrangement used in this study was designed to simulate natural color discrimination in the bluish-green region. We achieved this by chosing reflecting color discs with spectral reflection properties very similar to the natural colors of leaves and inanimate (anorganic) material like sand, ground, stones and by training free flying bees under natural daylight conditions. Therefore, we want to conclude that bees perceive colors in their choice behavior at the feeding place in such a way that the perceptual dimensions hue and saturation (or blue/greenness and UV/blue-greenness, respectively) are best suited to interprete their choice behavior. This suggests strongly that bees have a neural representation of color not basically different from our own subjective experience. Although it was shown with elegant experiments by Frisch (1914) that bees discriminate a blue color from a series of shades of bluish-greens which differ mainly in brightness, our study gives evidence to the first time that bees have true color vision with (own) perceptual dimensions other than the neural representations of differential excitations of the three spectral receptor types. Hue and saturation (or blue/greenness and UV/blue-greenness, respectively) are perceptual dimensions which are derived from the differential spectral input and its neural processing. Our analysis shows that not the spectral properties of the input directly but rather the derived dimensions best explain the color choice behavior in bees.

\section{Appendix I}

\section{Metric Multidimensional Scaling}

The metric model of Torgerson (1958) assumes a perceptual space to be Euclidean. The comparative distances $h_{i j}$ are expressed in this model as scalar products between every two vectors which represent the distance from the trained color to the tested colors $i, j$. For the compensation of measurement errors, the matrix of scalar products $\mathbf{B}^{\prime}$ of rankn (number of stimuli) is constructed such, that the zero point of the scale values of the stimuli, which will be calculated below, lies at the center:

$b_{i j}^{\prime}=\left(1 / n \sum_{i=1}^{n} h_{i j}^{2}+1 / n \sum_{j=1}^{n} h_{i j}^{2}-1 / n^{2} \sum_{i=1}^{n} \sum_{j=1}^{n} h_{i j}^{2}-h_{i j}^{2}\right) / 2$.

This implies, that Matrix $\mathbf{B}^{\prime}$ may have negative eigenvalues and that always one eigenvalue is zero.

The metric MDS procedure works direct with the metric distances $d_{i j}$ which are known up to an additive constant $c(5)$. The result of the procedure is sensitive on this constant. The iterative procedure of Messick and Abelson (1956) allows to calculate the additive constant $c$ from the eigenvalues of the matrix of scalar products $\mathbf{B}^{\prime}$ (7).

First, the elements of $\mathbf{D}$ are calculated from (5) with a trial value for $c$. The elements of $\mathbf{D}$ are taken as new comparative distances $h_{i j}^{\prime}$. The sum of the diagonal elements of $\mathbf{B}^{\prime}$ is calculated as:

$b_{j j}^{\prime}=n / 2 h_{. .}^{\prime 2}+n h_{.}^{\prime} . c+1 / 2(n-1) c^{2}$.

If $\mathbf{B}^{\prime}$ is of rank $d$, the same result is obtained from the sum of the $d$ largest eigenvalues $\beta_{m}$ (the remaining sum 
to zero) with help of the normalised eigenvectors $\mathbf{X}_{m}$ from:

$$
\begin{aligned}
\sum_{m=1}^{d} \beta_{m}= & \sum_{m=1}^{d}\left(\mathbf{X}_{m}^{T} \mathbf{U} \mathbf{X}_{m}\right)+c \sum_{m=1}^{d}\left(\mathbf{X}_{m}^{T} \mathbf{W} \mathbf{X}_{m}\right) \\
& +1 / 2 d c^{2}
\end{aligned}
$$

with

$u_{i j}=1 / 2\left(h_{i \cdot}^{\prime 2}+h_{. j}^{\prime 2}-h_{. .}^{\prime 2}-h_{i j}^{\prime 2}\right)$

$w_{i j}=\left(h_{i}^{\prime}+h_{\cdot j}^{\prime}-h_{. .}^{\prime}-h_{i j}^{\prime}\right)$

$v_{i j}=-1 / n$ for $i \neq j$ and $v_{i j}=1-1 / n$ for $i=j$.

The left sides of (8) and (9) are set equal to each other and the resulting quadratic equation is solved for $c$. The value of $c$ which gives the greater value for the left side of (9) is taken as a better value for the additive constant in (5). The iterative procedure converges quickly, two iterations are mostly sufficient. Matrix B, the scalarproduct matrix corresponding to the true distances, is now factorised by the method of principal components (Hotelling 1933); i.e., a $d \times n$ matrix of rank $d<n$ is desired, so that matrix $\mathbf{B}$ is reproduced by the matrix product of $\mathbf{A}$ and the transpose $\mathbf{A}^{T}$ :

$B \approx \mathbf{A A}^{T}$.

The columns of matrix $\mathbf{A}$ are the orthonormal scales in $d$ dimensions, which are calculated as the normalized eigenvectors of $\mathbf{B}$ times the square root of the corresponding eigenvalues (Überla 1982).

The goodness of reproduction can be checked in terms of the residual matrix $\mathbf{R}$ :

$r_{i j}=b_{i j}-\sum_{m=1}^{d} a_{i m} a_{j m}$.

The sum of the squares of the elements of the residual matrix $\mathbf{R}$ gives a global measure for the goodness of reproduction. The lowest number of dimensions for which the reproduction is good enough is taken as the dimension of the perceptual space. "Good enough" means, in this context, that an increase in dimension changes the goodness of reproduction less than a decrease. The model distances $\mathbf{D}$ are calculated from the scales with the Euclidean metric:

$d_{i j}=\left(\sum_{m=1}^{d}\left(a_{i m}-a_{j m}\right)^{2}\right)^{1 / 2}$.

The goodness of reproduction can be shown graphically by plotting the original proportions $\hat{\mathbf{P}}$ against the model proportions $\mathbf{P}(\mathbf{Z})$ derived from the model distances:

${ }_{k} z_{i j}=d_{k j}-d_{k i}$, where $\mathbf{D}$ are the distances from the trained color signal $k$ to the alternative ones $i, j$ and $\mathbf{Z}$ are the $z$-values of a triad of trained color signal $k$ and the tested pair $i, j$ of color signals.

\section{Appendix II}

Non-Metric Multidimensional Scaling

The non-metric model of Kruskal (1964a,b) overcomes the restriction of a Euclidean structure. It starts with a more general composition rule, the Minkowski or $p$-metric:

$d_{i j}=\left(\sum_{m=1}^{d}\left|a_{i m}-a_{j m}\right|^{p}\right)^{1 / p}$,

where $d_{i j}$ is the preceptual distance, the elements of $\mathbf{A}$ are the scales in $d$ dimensions, and $p$ is the Minkowski exponent. Important special cases of the Minkowski metric are: City-block metric $p=1$ (the absolute values of the differences on the scales are added). Euclidean metric $p=2$ (straight geometrical distance in Euclidean space). Dominance metric $p \rightarrow \infty$ (only the greatest difference on the scales contributes to the total difference).

The problem is, however, to find the minimal number of scales $d$, which allows a good reconstruction of the original distances. At the moment there exists no exact solution like Torgerson's for the problem of finding the scales with the more general metric. The problem of finding the additive constant $c$ is eliminated by normalising the scale values after each iteration step. That means, the stress function gives the relative difference in the rank orders of the measured distances and the model distances, which are not affected by an additive constant. Kruskal gave an iterative procedure, the method of steepest decent, which shall be used here. Starting from a random one, the configuration is modified step by step to minimise a goodness of reproduction function $S$ (normalised stress):

$S=\left(S^{\prime} / T^{\prime}\right)^{1 / 2}$

with

$$
\begin{aligned}
& S^{\prime}=\sum_{i<j}\left(d_{i j}-\hat{d}_{i j}\right)^{2} \\
& T^{\prime}=\sum_{i<j} d_{i j}^{2} .
\end{aligned}
$$

The new configuration $x^{\prime}$ is calculated from the old configuration $x$ by multiplication with the normalised gradient $g$, which gives the direction of steepest decent of the stress function in $d$ dimensions and a factor $a$, 
which controlls the step size.

$$
\begin{gathered}
x_{i m}^{\prime}=x_{i m}+a g_{i m} /\left(1 / n \sum_{i, m} g_{i m}^{2}\right)^{1 / 2} ; \\
i=1, \ldots, n ; \quad m=1, \ldots, d \\
g_{i m}=S \sum_{i, j}\left(\delta^{i l}-\delta^{i k}\right)\left(\left(d_{l k}-\hat{d}_{l k}\right) / S^{\prime}-d_{l k} / T^{\prime}\right) \\
\cdot\left|x_{l m}-x_{k m}\right|^{d-1} / d_{l k}^{d-1} \operatorname{signum}\left(x_{1 m}-x_{k m}\right)
\end{gathered}
$$

$\delta^{i l}$ are the Kronecker symbols $\left(\delta^{i l}=1\right.$ if $i=l, \delta^{i l}=0$ if $i \neq l$ ). The procedure has to be repeated many times, with different starting configurations because of convergence to local minima of $S$. For details of the algorithm see Kruskal $(1964 a, b)$. Calculation time is saved when the metric solution is taken as starting configuration which should already be close to the absolut minimum of the normalised stress $S$.

\section{References}

Ahrens HJ (1974) Multidimensionale Skalierung. Methodik, Theorie und empirische Gültigkeit mit Anwendungen aus der differentiellen Psychologie und Sozialpsychologie. Beltz, Weinheim

Backhaus W, Menzel R (1984) Multidimensional scaling of the colour space of honeybees. Verh Dtsch Zool Ges 77:230

Backhaus W, Menzel R (1987) Color distance derived from a receptor model of color vision in the honeybee. Biol Cybern $55: 321-331$

Daumer K (1956) Reizmetrische Untersuchung des Farbensehens der Bienen. Z Vergl Physiol 38:413-478

Davison ML (1983) Multidimensional scaling. Wiley, New York

Frisch K v (1914) Der Farbensinn und Formensinn der Bienen. Zool Jb Abt Allg Zool Physiol 35:1

Guilford JP (1937) Scale values derived from the method of choices. Psychometrika 2:139-150

Helm CE (1964) Multidimensional ratio scaling analysis of perceived color relations. J Opt Soc Am 54:256-262

Helversen O v (1972) Zur spektralen Unterschiedsempfindlichkeit der Honigbiene. J Comp Physiol 80:439-472

Hotelling H (1933) Analysis of a complex of statistical variables into principale components. J Educ Psychol 24:417-441, $498-520$

Indow T, Ohsumi K (1972) Multidimensional mapping of sixty Munsell colors by nonmetric procedure. In: Vos JJ, et al. (eds) Color metrics. AIC, Holland

Krantz DH (1967) Rational distance functions for multidimensional scaling. J Math Psychol 4:226-245
Kruskal JB (1964a) Multidimensional scaling by optimizing goodness of fit to a nonmetric hypothesis. Psychometrika 29:1-29

Kruskal JB (1964b) Nonmetric multidimensional scaling: A numerical method. Psychometrika 29:115-131

Kruskal JB, Wish M (1978) Multidimensional scaling. Sage, Beverly Hills, CA

Labhart T (1974) Behavioral analysis of light intensity discrimination and spectral sensitivity in the honey bee, Apis mellifera. J Comp Physiol 95:203-216

Lieke E (1987) Honeybees have a perceptual dimension of color saturation. J Comp Physiol (in press)

Menzel R (1967) Untersuchungen zum Erlernen von Spektralfarben durch die Honigbiene (Apis mellifica). Z Vergl Physiol $56: 22-62$

Menzel R, Greggers U (1985) Natural phototaxis and its relationship to colour vision in honeybees. J Comp Physiol 157:311-321

Menzel R, Lieke E (1983) Antagonistic color effects in spatial vision of honeybees. J Comp Physiol 141:389-393

Nash JC (1979) Compact numerical methods for computers. Hilger, Bristol

Messick SJ, Abelson RP (1956) The additive constant problem in multidimensional scaling. Psychometrika 21:1-17

Sixt1 F (1982) Meßmethoden der Psychologie. Theoretische Grundlagen und Probleme. 2. Edn. Beltz, Weinheim

Thurstone LL (1927) A law of comparative judgment. Psychol Rev 34:273-286

Torgerson WS (1951) A theoretical and empirical investigation of multidimensional scaling. Ph.D. Thesis, Princeton University

Torgerson WS (1958) Theory and methods of scaling. Wiley, New York

Überla K (1982) Faktorenanalyse. Eine systematische Einführung für Psychologen, Mediziner, Wirtschafts- und Sozialwissenschaftler. 2. Edn. Springer, Berlin Heidelberg New York

Werner A, Menzel R (1984) Color discrimination and receptor color space in honeybees. Verh Dtsch Zool Ges 77:229

Zielinski R (1978) Erzeugung von Zufallszahlen. Programmierung und Test auf Digitalrechnern. Deutsch, Frankfurt/Main

Prof. Dr. R. Menzel

Freie Universität Berlin

Fachbereich Biologie, WE5

Neurobiologie

Königin-Luise-Strasse 28-30

D-1000 Berlin 33

Germany 